\title{
REDUCING FLOODING OF ZONE ROUTING PROTOCOL IN MOBILE AD-HOC NETWORKS
}

\author{
ISHAQ AHMAD $^{1}$, OMAR M. BARUKAB ${ }^{2}$ \\ ${ }^{1}$ Department of Computer Science, Abdul Wali Khan University Mardan \\ ${ }^{2,3}$ Faculty of Computing and Information Technology - Rabigh, King Abdulaziz Univeristy, \\ Saudi Arabia. \\ ishaqictm@gmail.com, obarukab@kau.edu.sa
}

\begin{abstract}
Revised March 2016
ABSTRACT. Mobile ad-hoc networks (MANETs) are temporary networks without any pre-existing infrastructure in which nodes are connected through wireless channels. There is no centralized administration in this network because nodes are not fixed at their locations, they are continuously moving to different locations. Each node in MANETs, has dynamic capabilities because at one point acts as a host while in some other time becomes a router which can forward messages on the network to other nodes. One of the complicated issues in MANETs is routing due to their dynamic topology which is frequently changes due to mobility of nodes. In order to forward packets in this complex topology using optimal routes there is a need for efficient routing protocols. There are three categories of routing protocols in MANETs named as reactive, proactive and hybrid respectively. We have used a hybrid type protocol named Zone Routing Protocol (ZRP) for routing in our research. In order to control unwanted flooding in the overlapping zones of ZRP we have proposed selective bordercasting technique for route discovery in. In this technique route discovery exchange messages are highly reduced in the outer zone of the ZRP.
\end{abstract}

Keywords: MANET, Routing, Flooding, Broadcasting, Proactive, Reactive, Hybrid, ZRP, IARP, IERP.

1. Introduction: Ad-Hoc network basically is a type of network connection that needs at least two Wi-Fi devices or wireless local area network cards for its establishment. A Mobile Ad-Hoc Network (MANET) is a distributed network having independent moving nodes. These nodes are able to communicate with each other without the need of any physical media [20]. Due to moving nodes in the Mobile Ad-Hoc network, the physical infrastructure of the network may rapidly be changing. Thus it is difficult and hard to use conventional static routing tables to cope with this dynamic environment [6]. Instead, the requirement of every node here is to select with high accuracy the optimal path towards the destination. Because here the layout is continuously changing and thus a great care is required while selecting the routes among the various routes. MANET offers many advantages over single hop networks. Transmission power and energy is efficiently used in MANET because of its transmission on shorter links. MANET utilizes the transmission medium very efficiently due to which it offers high data rate and throughput. It also eliminates the need of cables, which reduces the extra cos [4].

Routing protocols in MANET [23] are based on the infrastructure of internetwork protocol (IP). When we are going to create an Ad-Hoc network, we simply take at least two Wi-Fi devices then go to the network setting and sharing section to create Ad-Hoc connection with a different levels of security. Then, we setup an IP for the very similar Ad-Hoc connection. Then we go to the next wireless device and configure it a similar way. So with this process an efficient Ad-Hoc network come into existence with at least $54 \mathrm{mbps}$. It may perform its operation by using unicast, multicast or hybrid techniques due to which it permits for communication regardless of considering it as a different entity on using the wired IP services as a standard. A 
thorough approach discusses the complexity of various phases of Mobile Ad-Hoc networking is discussed in [4]. Figure 1 depicts different types of routing protocols.

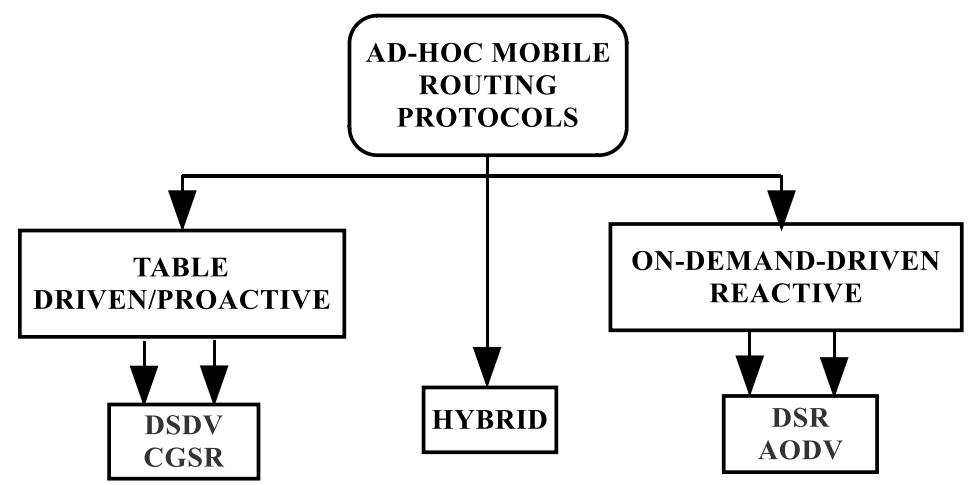

Figure 1: Hierarchy of Routing Protocols.

There are a number of protocols that are needed for the establishment of the Ad-Hoc network. Various number of routing protocols are established by the Internet Engineering Task Force (IETF) which is an effective working group for making and investigating routing protocols which are defined in $[3,10,15,24]$ for MANET. These protocols can generally be divided/categorized into reactive [2] and proactive protocol groups. A comparison between the two types of protocols is shown in Table.

Proactive routing protocols established pre-defined path before the data packets forwarded from source to destination node. These protocols update the topology of the network constantly, and in this mechanism every node knows about all other nodes on the network in advance. Proactive routing protocols are implemented to maintain the latest route information of the whole network layout. According to this preview, the path is determined and immediately presented when a message is required to be communicated. It uses the same method which is implemented in the IP-based wired networks [5]. For example, in an Open Shortest Path First protocol (OSPF) or Pro-active protocols that uses this type of technique, which is very much similar to the wired network routing protocols. New routes can be determined by regularly performing processing on the available routes; as a result, latest topology of the network is maintained. It gives a node the ability of effectively performing packet forwarding as it has known routes on the same occasion when message reaches to the node. These protocols (table driven) are also known as Pro-active routing protocols.

Proactive protocols are referred to as link state or distance vector protocols. Due to node's mobility, they keep the dynamically changing topology up to date continuously. They consume the available bandwidth which is a limited resource in such ad-hoc environment. These routes exist only for a very limited periods of time. The class of Distance-Vector protocols, along with Destination-Sequenced Distance-Vector Routing (DSDV) [22] belongs to the class of table-driven protocols. Destination Sequenced Distance Vector protocol (DSDV) [21] is a table-driven proactive protocol, which is based on the distributed Bellman-Ford routing algorithm in which each node contains routing tables, which store all the related information of hops in the network and the entire respective path between nodes. Each node keeps all the routing information of the network in their routing table, this routing table consist of all of the information about routes in the network. When nodes uses proactive routing protocol each node exchanges topology information to one another in a predefine manner. The Optimized Link State Routing Protocol (OLSR) [7, 9, 30] floods or broadcasts packets throughout the network, every node capture these broadcast packets, update their routing table and then rebroadcast them. These packets contains a sequence number which avoids loop or duplication of the packet in the network [8], but only Multi Point distribution Relays (MPR) nodes can broadcast route packets, these MPR nodes are selected within the surrounding range of a source node.

Bandwidth utilization of the proactive protocol is high for maintaining information about paths, while in case of reactive approach the problem is the latency in path request. Path searching through reactive approach is costly because it performs flooding for this purpose [18]. Hybrid protocol [21] is the strength of both proactive and reactive protocols. Using hybrid protocol; the network is divided into Zones. One type of routing protocols is used in the one zone and different routing protocols are used in different Zones. Zone 
Routing Protocol [12] is an example of hybrid protocol. The zone routing protocol objective is to overcome the limitations by merging the best characteristics of dual platforms into one framework. Proactive routing protocols and reactive routing protocols are used in combination to perform the implementation of routing process in MANET which have their own limitations. The zone routing protocol which is discussed here benefits from the two approaches [12] in which it combines proactive and reactive approaches into one scheme and getting the complete benefits of proactive detection inside the neighborhood of node, and the exchange of messages in the neighborhoods which is carried out by reactive protocols. Communication between nodes in MANET occurs mostly when they are neighbors to each other. Topology variations are considered essential in the locality of a device. The insertion or the exclusion of a node has a very low impact on the node neighborhood in the network. ZRP is the combination of proactive and reactive protocol features; therefore the feature of ZRP is to reduce the latency in route discovery and to reduce the number of control messages during communication due to which flooding in the network are reduced. In a network, each node creates their own zone which is also called neighborhood of that specific node, and nodes within the zone of nodes are the neighbors and the remaining nodes which are out of zone are known as the peripheral nodes [15].

As explained earlier, that ZRP is a sketch or platform for other protocols and it is just a framework not a complete protocol like others. The split-up of network nodes into local neighborhood is global. Zone name is given to this process because of the local interaction and relations of nodes where each node may be within multiple overlapping zones, and the size of the zone is different [11]. Size of the zone is not available in advance that is required, but the length $\mathrm{p}$ of radius is given, in which $\mathrm{p}$ is the amount of distance of the device to the whole zone in terms of hop counts. Network is distributed into overlapping, variable-sized zones; hierarchical map of the network is avoided in ZRP while extra load is included in maintaining the network topology map. Otherwise the network structure is considered flat, and in case of the identification of overlapping zones there is a possibility of route optimization. While the impression of zones often seems to compare the same nature of the services of a telephone network. It is worthy point that is to be noted that every device in the network contains a proper routing zone, and is independent on constant nodes.

\subsubsection{Architecture of Zone Routing Protocol}

Zone Routing Protocol plays an important role in the minimization of flooding in MANET. In fact Zone Routing Protocol has several elements which makes the ZRP more optimized for providing better performance and reducing flooding in MANET. The components of ZRP are working freely in routing environment. These elements depend upon the zone of the nodes, because some components perform their task inside the zone of the node while others are working outside of the zone of source node for the discovery of route from source to destination node. ZRP consists of three sub- protocols (components). The architecture of the ZRP is depicted as shown in figure 2. It is clear from the figure that Zone routing protocol contains many architectural portions, which work in collaboration with each other to offer complete benefits of routing to ZRP. Every architectural portion of ZRP operates autonomously from other portion and due to this reason they are not bound to use the same technologies that can exploit performance in its specified location [20]. 

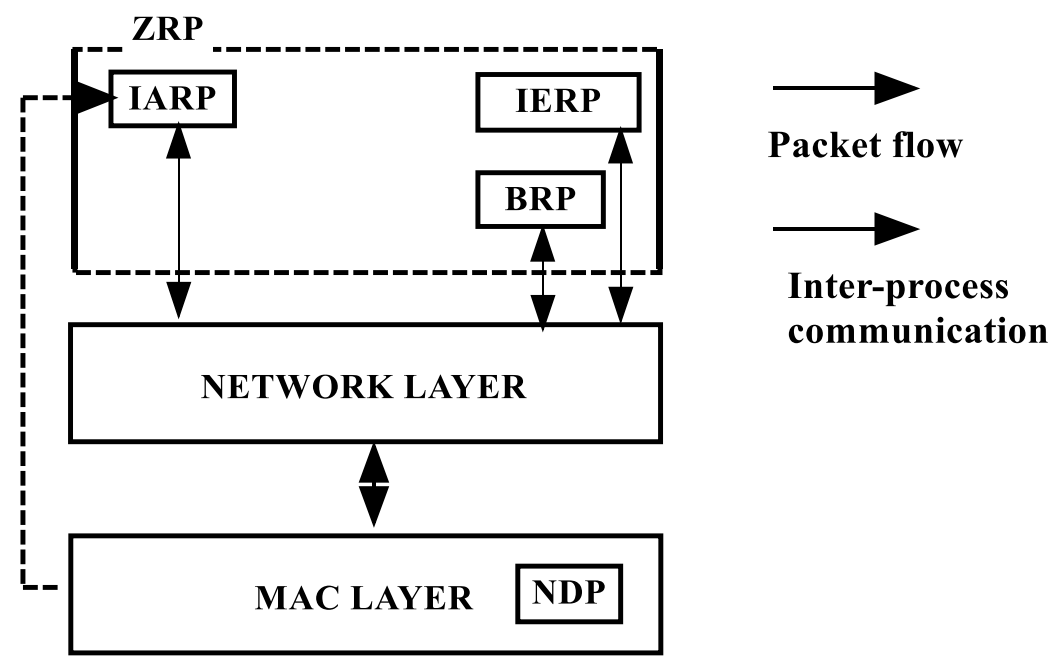

Figure 2. The ZRP Architecture.

If we consider protocols from reactive approach that is, DSR or AODV [18] can be selected as the Intra-zone routing protocol IARP while for IERP we can select protocols from proactive approach such as DSDV or OLSR [13]. ZRP nature shows that it belongs to hierarchical category but it is important to highlight that ZRP belongs to the flat category of routing protocols. Different protocols are taken in the hierarchical structure of the ZRP in order to show communication between:

a. Every separate node in the cluster of nodes is within the same zone and

b. Different clusters are among different zones.

Dissimilarity is very clear here in ZRP because of the correspondence of nodes and zones is one to one which results in the overlapping zones retained separately by every node individually [12].

1.3.2 Intra-Zone Routing Protocol (IARP)

Local path searching component of the ZRP is called intra zone routing protocol (IARP). Neighbour Discovery Protocol (NDP) is used on the link layer due to which the local path searching of nodes is carried out. In order to make conversation, a node with its interior node belonging to its zone in the IARP is used for this purpose and it is controlled by the radius of zone $\mathrm{p}$ which is the amount of distance in terms of hop count from peripheral nodes. To discover local neighbours, IARP uses NDP. It uses it to communicate with neighbour nodes which are located in the same zone [12]. Since in MANET nodes are frequently change their positions, it may have immense impacts on routing. Size of zone and IARP scope are equal. Time to live (TTL) is used during the updating of routing table by IARP. When a requested message travels from sender device to its neighbour nodes, TTL automatically decreases. TTL becomes zero as border device receives the requested message. Thus the local neighbourhood of a node may change very rapidly and as a result changes also appear in the topology locally which is likely to have a greater impact on a node's routing behaviour than other changes on the other side of the network. The IARP is also called proactive, table-driven protocol. All the information about the local routes are maintained by this protocol which is based on the periodic exchange of neighbour discovery messages.

Refreshing of routes in the routing table is required so that every node can maintain the latest route information in it routing table about its local neighbours. The IARP is responsible for finding out routes towards its local neighbours and to delete the idle links in addition to the replacement of a route with the shorter ones in terms of hops that are detected. IARP bypasses broken links in a network containing large number of nodes which is a big advantage in route flooding.

1.3.3 Inter-Zone Routing Protocol (IERP)

The universally reactive routing module is named inter-zone routing protocol (IERP). It is the reactive strategy used by ZRP in mobile ad-hoc networks for routing between zones, get the benefits of the predefined zone of the node's local topology and, apply a reactive approach to make the conversation possible between nodes belonging to different network zones. Thus the Inter-Zone Routing protocols are uses for the 
communication among different Zones.

Requests for paths inside IERP are allocated reactively which are only made when a path is to be requested. The duration results in the path searching (contrary to IARP, in which path are immediately presented) is decremented with the help of broadcasting, a method where device does not send request to all of the other local devices, but just submitted to the nodes in its proximity. Moreover, a device is unable to exchange a query in response to those devices from which it has received a query message, even if there are peripheral nodes.

In order to transform a current methodology used in ZRP for using IERP as reactive approach. Native paths Updates are disabled by proactive approach which is implemented through IARP. Moreover, information which is collected by IARP is further used by IERP in order to know about the local paths. It also uses this information to identify the method by which the path has been changed. Bordercast Resolution Protocol (BRP) is used for forwarding route request message to the neighbour nodes instead of using flooding [23].

\section{Literature Review}

In the ZRP protocol, nodes inside the routing zone area are called neighbour nodes for the source node while these neighbour nodes that are the nearest nodes in the zone of the source node are called routing zone nodes. The distance of the source nodes is less than the radius of the routing zone node. Commonly the neighbour nodes are at one hop away from the source node and normally the radius of the routing zone node is greater than the hop count of the neighbour nodes. The peripheral node distance is equal to the radius of the routing zone node. These nodes are at the border of the source node. When source node is going to communicate with the destination node and this destination node is out from the zone area of source node then it needs to discover the route for the destination node. The neighbour nodes are accessed by IARP of ZRP. In the work of [12] the authors have discussed schemes for controlling query which effects routing zone structure in order to improve the performance of queries overlapping in term of prevention and detection. These methods can be applied to one or several channels in the network to enhance ZRP performance in both latency and traffic control. Methods that we have used for controlling the query permit ZRP to give paths to every node which is reachable in a network with minimum traffic control as compared to the discovery of reactive paths or like the link state proactive, and with minimum delay as compared to conventional flood searching mechanism. In [25] authors have discussed that ZRP protocol has two parts i.e. Intra Zone Routing protocol (IARP), which work locally using table driven technique and the second one is the inter-zone routing protocol, which works globally on demand. The main focus of this work was on finding the initial route discovery delay and throughput for performance evaluation issues without giving any consideration to flooding. In the paper by [27] DSR protocol of IERP is compared with other routing protocols which are used to discover and maintain the route. It is very useful for low mobility network nodes. Broadcasting is done by the node to all those nodes again which have been covered by the request of the discovery route. Nodes which have already received the request query message have the ability to discard such request packet. Early termination is the feature of nodes which check their routing table for such a request. Therefore, it provides help to control the node traffic in the zone. In work by [19] authors have discussed Zone routing protocol that it plays an important role in the mobile ad hoc networks. Although the routing protocol is facing a number of challenges such as the topology of the network changes with continuous rate, and the routing protocols have less power of transmission. ZRP is not more significant in some circumstances. The ZRP is the combination of proactive routing protocols and reactive routing protocols advantages such as table-driven and on demand routing. Table-driven protocols keep the information of the entire routing zone which have maximum bandwidth and keep extra information about nodes. Although on demand protocols have removed this maximum bandwidth problem but create high delay rate for route discovery. Therefore, our implementation of ZRP is trying to reduce such a problem in the MANETs up to more extent.

Researchers in [2] discussed the structure and advantages of the Dynamic Source Routing Protocol (DSR) protocol which is based on two mechanisms i.e. broadcasting and eavesdropping. The routing zone node broadcast the request query to each node, checks the query message if it has already covered it or has come for the first time to the routing table. These overhearing's are only notified and control the maximum traffic on the zone. Many routing zone nodes sends the route discovery request many times to other nodes, when each node starts such type of request, it creates flooding in the network. When one node broadcast the request message to every other nodes including neighbouring nodes which receive this request for the first time then it is efficient, but when every node starts sending unwanted traffic then it creates flooding problem 
in the network. Researchers in [14] authors have evaluated ZRP protocol which is a hybrid routing protocol of MANETs and consists of both IARP and IERP protocols. ZRP provides good framework to the MANETs and reduces flooding up to some level but this research work does not give optimum zone radius. Proactive routing protocols work within the routing zone which produces routing tables and some on demand protocols are used to minimize delay and latency rate and also minimize overhead information on nodes. Next, in the work of [17] authors have discussed evaluation and enhancements of ZRP which provides maintenance procedure for failed links but still it is a framework that lacks more efficient implementation. Authors in [22] have evaluated Destination Sequence Distance Vector protocol (DSDV), in which each node forward the routing table information periodically to neighbour nodes. The route which is preferred for routing has greater sequence number, when destination is reached then it advertises new sequence number. Each node maintains routing table for itself and keeps information like next hop, cost, and metric of destination side but this approach consume more bandwidth and high overhead. In the work accomplished by $[26,28]$ shows that the performance evaluation of the Temporally Ordered Routing Algorithm (TORA), Optimal Link State Routing protocol (OLSR), DSR and the Ad-hoc On-demand Distance Vector routing protocol (AODV) is carried out with the help of simulation in different network scenarios i.e. different network sizes, traffic rush and node mobility. Simulation results revealed that the performance of TORA in long and average network sizes is better than as compared to other protocols under heavy traffic. When the network size is small and movement of node is low then DSR is a best choice in such environment. DSR performance is also good when network size is large and movement of nodes are fast. When the traffic load is high and the network size is moderate then in such situations AODV performance is efficient. When OLSR is evaluated, it is observed in many cases that it performs well as compared to other protocols but problems with OLSR occurs when node movement becomes fast and network traffic increases. In the work done by [29] OLSR and AODV performed better than TORA in terms of throughput since in this parameter it performs degraded. The decision of the route in AODV is dependent on the distance which is obtained in reply of the destination sequence number. Sequence number is also used by Link Durability Routing protocol (LDR) but destination node control it. The arrangement of nodes is performed on the basis of labels which are assigned to each destination. This labelling technique gives the facility of loop free environment which is the combination of sequence number and distance. In the former work, random waypoint model is used as the mobility model having different pause times. The performance of these protocols are evaluated on the basis of packet delivery ratio, end to end delay, hop count based on Time to Live (TTL) in which AODV performed better than both ZRP and DSR [1, 11, 19]. The performance of AODV and DSR is better than ZRP in case of packet delivery ratio. ZRP, LAR, DSR, and AODV were analysed under different network sizes in mobile ad-hoc networks [16]. The performance of all these protocols shows better results when the size of the network is small. Packet delivery ratio in bigger networks is observed in which AODV outperformed both DSR and ZRP protocols.

Performance Metrics and Simulation Setup

We have used four metrics for the performance evaluation of our routing protocol ZRP. For this purpose, we have considered packet delivery ratio, packet's drop rate, average end-to-end delay and average throughput as our metrics. We will make our selection defendable based on the above mentioned criteria. These metrics are described as follows:

\subsection{Packet Delivery Ratio}

Packet Delivery Fraction is the ratio of the total number of CBR received packet by total number of sent packet by CBR. When the radius of zone routing is in its lower value then the packet delivery ratio is at its possible maximum speed, but when the radius increases, the packet delivery is come down to its lower level. This ratio is generated by Constant Bit Rate (CBR) towards the destination of packet transmission, and Packet Delivery Fraction (PDF) shows that how much packets are successfully reached from source to destination. Therefore the maximum value of PDF gives better result because the greater the value of PDF shows that the maximum number of packets are successfully received by the destination node. If the packet delivery ratio is small, this means that larger number of packets are dropped on the way so the results of PDF will demonstrate how the protocol is efficient and reliable.

Packet Delivery Ratio $=($ total received data packets / total data packets sent $)$ or $\mathrm{PDF}=($ Total number of CBR pkt Received $/$ Total CBR pkt sent $) \times 100$

$$
\text { Packet delivery ration }=\frac{\sum \text { CBR packets received by CBR sinks }}{\sum \text { CBR packets sent by CBR sources }}
$$




\subsection{Drop Rate of Packet}

This parameter represents the number of packets which any mobile node was dropped during the discovery of the shortest path for packet forwarding from source to destination, because when the number of packets forwarded toward a destination, some packets are lost in the way or from the queue of the node. The following command is used for capturing the dropped packets.

\subsection{Average End-to-End Delay}

$$
\text { \#grep “^d" simple.tr | grep "CBR” | grep “_ | _ RTR > }
$$

This mechanism defines the average possible delay in data packets sending destined to a node. This endto- end delay may be route discovery delay, buffering delay, retransmission delay, or latency. The performance of the network is also depends upon the average end-to-delay. The following equation is used for finding the average end- to-end delay.

$$
\text { Average end }- \text { to }- \text { end Delay }=\frac{\text { No. packet receive time }- \text { No. packet send time }}{\text { Total number of packet received }}
$$

OR

$$
\text { Average end to end delay }=\sum \frac{\text { time received }- \text { time sent }}{\text { total data packets received }}
$$

OR

$$
\text { Average end }- \text { to end delay }=\frac{\sum \mathrm{CBR}_{\text {sent }}-\left(\mathrm{CBR}_{\text {rec }} \text { Time }\right)}{\sum \mathrm{CBR}_{\text {rec }}}
$$

In performing route discovery source node broadcast the request query to each node on the network, such type of delay on the network is called route discovery delay. The efficiency of the network will degrade if the source node retransmits packet on the route toward the destination node. Therefore, it will affected the performance of the protocol's performance, because it takes more time for receiving packets.

3.4 Average Throughput: Throughput is defined as the ratio of the number of received packets by the destination node to the number of the transmitted packets by the source node. The average throughput will increase when the radius of the zone is small, because in smaller zone the number of nodes is less as compared to a zone with larger radius.

3.2 Simulation Setup:The following set of parameters are used in this research study which are: Area of the network is chosen to be $1000 \mathrm{~m} \times 1000 \mathrm{~m}$, while link bandwidth is $1 \mathrm{Mbps}$. The simulation time is set 400 seconds, and the traffic type is CBR which is commonly used in MANET. Packet size is set to 128 bytes and the maximum data rate is 2 bytes per second. Finally, a radius of the routing zone is selected within a range from 1 to 10. These parameters are tabulated as shown in table 1. The performance of the Zone Routing Protocol (ZRP) is carried out in a mobile ad-hoc Network environment. Figure 3 depicts a snapshot of the simulation environment.

Table 1 Network Parameters.

\begin{tabular}{|l|c|}
\hline PROTOCOL & ZRP \\
\hline Area of Network & $1000 \mathrm{~m} \times 1000 \mathrm{~m}$ \\
\hline Number of Nodes & 30 \\
\hline $\begin{array}{l}\text { MAX Speed (Data } \\
\text { Rate) }\end{array}$ & 16 bits/Sec \\
\hline Packet Size & 128 bytes \\
\hline Simulation time & 400 Sec \\
\hline Traffic Type & $\begin{array}{c}\text { Constant Bit Rate } \\
\text { (CBR) }\end{array}$ \\
\hline Bandwidth & 1Mbps \\
\hline Node Placement & Random \\
\hline
\end{tabular}




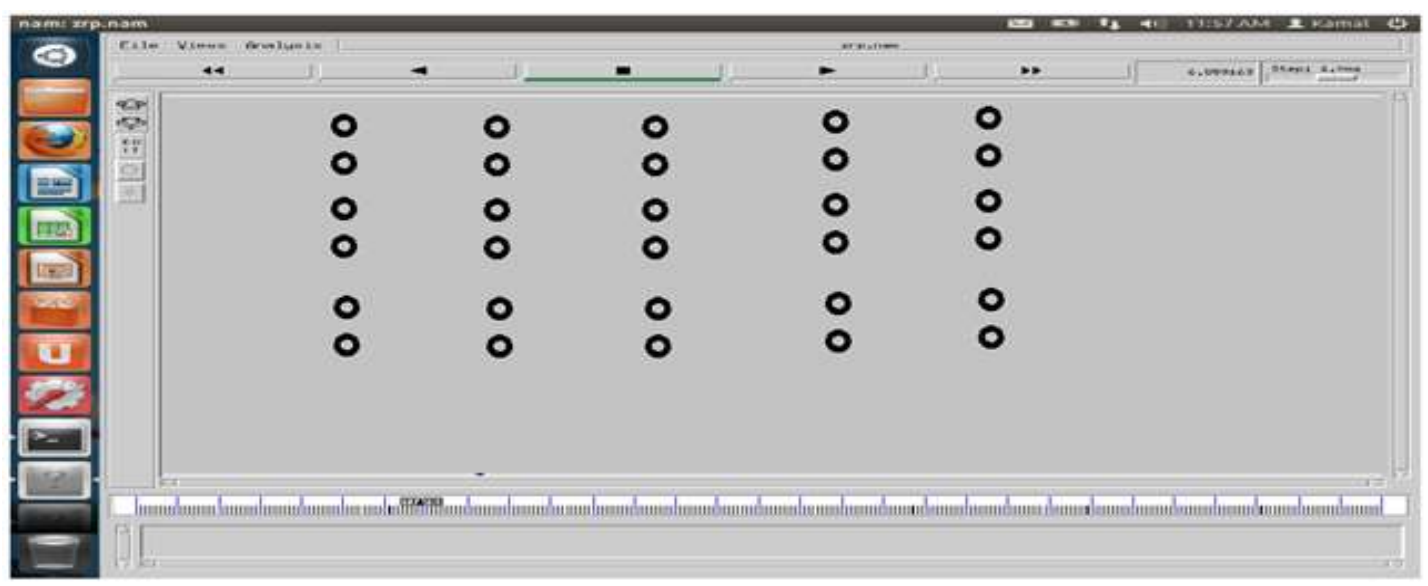

Figure 3. Screenshot of Simulation Environment

If the traffic is increased in the proactive IARP and the traffic of IERP is decreased in some way, then this scenario is referred to undershoot in the optimum zone radius, but when the traffic is decreased in the IARP and increased in the IERP then it creates "overshoot" in the routing radius. The possible optimization is requiring that the ratio of both (IARP \& IERP) is close to each other up to a maximum level. Routing is a very important component of MANET, because the topology is continuously changing, therefore the path from source to destination node is also changes according to the network topology. When the speed and the number of nodes are increased, the average end-to-end delay is also increased because the route finding process becomes very difficult in this high mobility environment. The speed of the ZRP not only caused to drop a large amount of packets but also increases the delay and routing overhead. If the numbers of nodes are increased in case of ZRP protocol, the packet delivery ratio is deceases. Therefore ZRP is more efficient for large size networks and effective in the zone with optimal radius. Actually ZRP has three elements namely IARP, IERP and the Broadcast Resolution Protocol (BRP), which is commonly depends on the radius of nodes in the network, because when the radius of the node is optimal it can reduce the routing traffic and improves the performance of ZRP. Figure 4. (a) Illustrates the last previous updated work in which packet delivery ratio of ZRP in comparison with other protocols in percentage with various pause times. As we can see from the simulation results that the packet delivery ratio of ZRP with traditional flooding for route discovery and our proposed selective bordercasting technique for route discovery in ZRP is equal even our proposed technique has reduced route request messages up to $50 \%$ without affecting the delivery ration which a tremendous enhancement is shown in figure 4. (b).

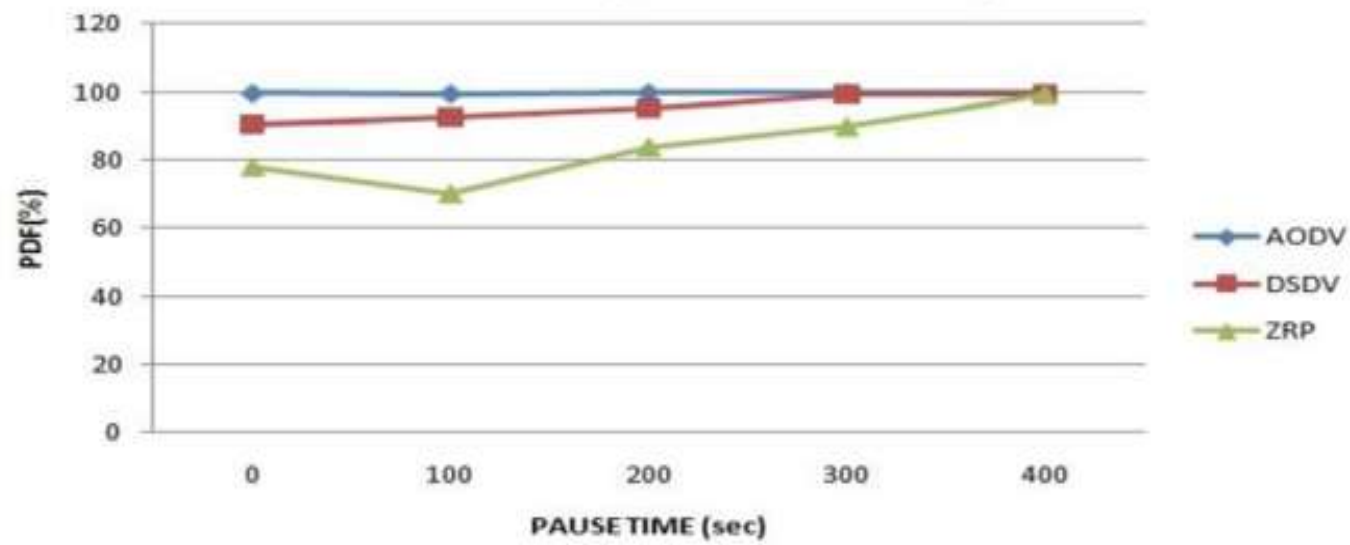

Figure 4. (a): Packet Delivery Ratio of ZRP (Previous Work). 


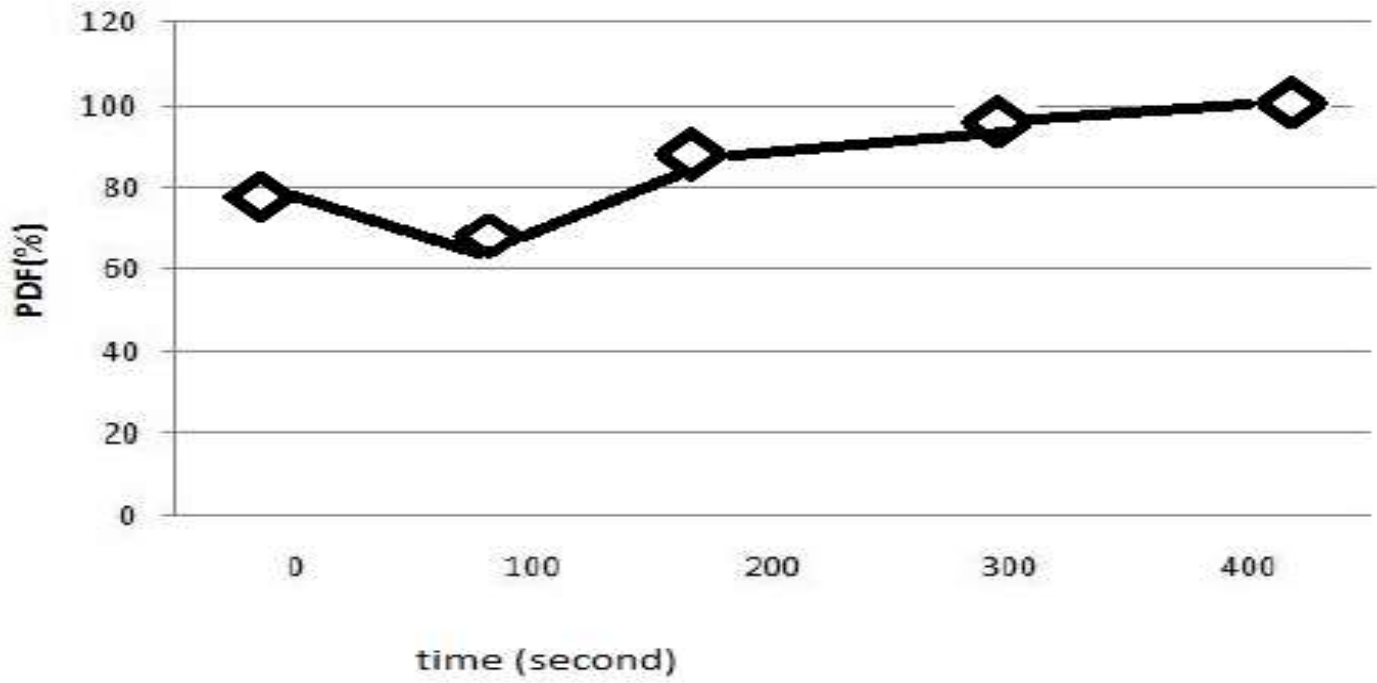

Figure 4. (b): Packet Delivery Ratio of ZRP (Proposed Work).

The packet delivery is increasing with time ( $0 \mathrm{~s}$ to $400 \mathrm{~s}$ ), in this case more packets are reached from the source to the destination node and a small amount of packets are dropped before received by the receiving node. Routing table of a node is populated with routes information that reduces flooding for route requests in the network. The performance of ZRP is increases with increasing the time component, because the delivery of packet is larger with time, and the average end to end delay, and dropping rate of packets are decreases continuously. The large value of throughput shows that the performance is increases with time of ZRP. So in the packet delivery ratio the maximum performance of the Zone Routing Protocol is $97 \%$ because a large number of packets are received on the network and end-t- end delay is decreased at this level. Table 2 below shows the packet delivery ration.

Table 2: Packet Delivery Ratio.

\begin{tabular}{|c|c|c|c|c|c|}
\hline ZRP & $\begin{array}{c}\text { Total } \\
\text { Routing } \\
\text { packets }\end{array}$ & $\begin{array}{c}\text { Received } \\
\text { data } \\
\text { packets }\end{array}$ & $\begin{array}{c}\text { Average } \\
\text { end to end } \\
\text { delay (sec) }\end{array}$ & $\begin{array}{c}\text { Dropped } \\
\text { from Queue }\end{array}$ & Throughput \\
\hline Os & 155821 & 9254 & $\mathbf{0 . 0 9 8}$ & $\mathbf{5 3 2 6}$ & $\mathbf{1 3 6 2 4 . 0 1}$ \\
\hline $100 s$ & 151405 & 8951 & $\mathbf{0 . 0 8 7}$ & 6784 & 13215.32 \\
\hline $200 s$ & 148943 & 10861 & $\mathbf{0 . 0 6 8}$ & 4586 & 14832.97 \\
\hline $300 s$ & 150747 & 11064 & $\mathbf{0 . 0 4 5}$ & $\mathbf{3 8 0 0}$ & 15462.76 \\
\hline $400 s$ & 142395 & 12584 & $\mathbf{0 . 0 1 9}$ & 1105 & 17952.85 \\
\hline
\end{tabular}

The average end-to-end delay metric of our proposed technique work is reduced up to a large extent as it is clear from both simulations shown in figure 4. (C) and 4. (d). It is increasing when the number of nodes is increased, although the throughput is increases but the dropping ratio of packet is also move upward with end to-end-delay and the performance of the ZRP is slightly lower as compared to other models. Table 3 shows the average end-to-end Delay. 


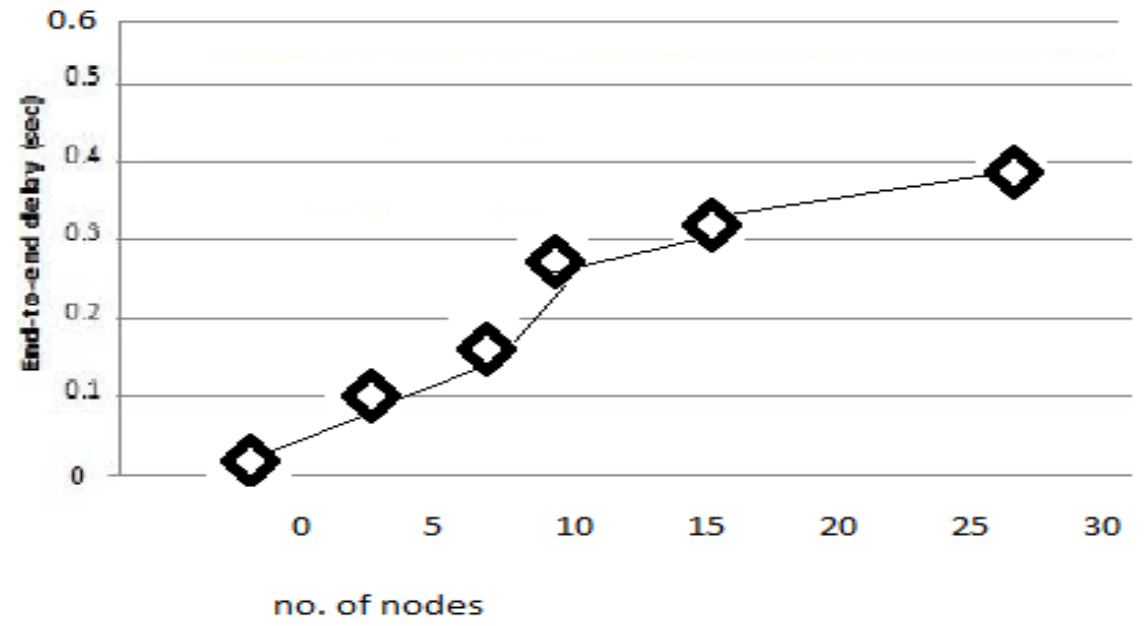

Figure 4. (c): Average End To End Delay of ZRP (Proposed Work).

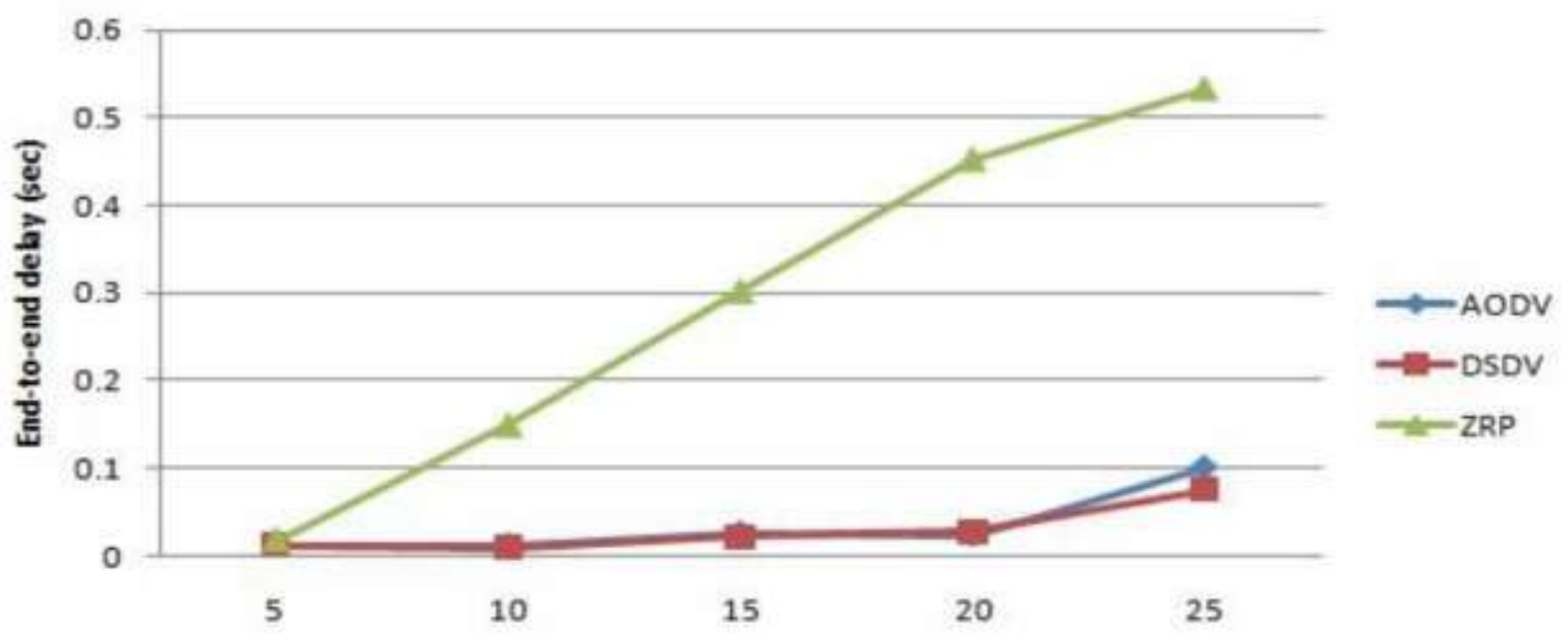

Figure 4. (d): Average End to End Delay of ZRP (Previous Work).

Table 3: Average End-to-end Delay.

\begin{tabular}{|c|c|c|c|c|c|}
\hline ZRP & $\begin{array}{c}\text { Total } \\
\text { Routing } \\
\text { packets }\end{array}$ & $\begin{array}{c}\text { Received } \\
\text { data } \\
\text { packets }\end{array}$ & $\begin{array}{c}\text { Average } \\
\text { end to end } \\
\text { delay (sec) }\end{array}$ & $\begin{array}{c}\text { Dropped } \\
\text { from Queue }\end{array}$ & Throughput \\
\hline $4 \mathrm{pkt} / \mathrm{s}$ & 150230 & $\mathbf{8 9 6 5}$ & $\mathbf{0 . 1 5}$ & 10597 & $\mathbf{1 3 4 6 4 . 2 3}$ \\
\hline $8 \mathrm{pkt} / \mathrm{s}$ & 163521 & 9958 & $\mathbf{0 . 2 9 9}$ & 25911 & $\mathbf{1 4 9 8 5 . 6 2}$ \\
\hline $12 \mathrm{pkt} / \mathrm{s}$ & 175295 & 10753 & $\mathbf{0 . 3 2 2}$ & 40258 & $\mathbf{1 5 1 3 6 . 9 1}$ \\
\hline $16 \mathrm{pkt} / \mathrm{s}$ & 188512 & 11096 & $\mathbf{0 . 3 8 1}$ & $\mathbf{5 5 7 6 8}$ & $\mathbf{1 6 9 8 5 . 7 2}$ \\
\hline
\end{tabular}

In our proposed work the ZRP throughput which is shown in figure 4. (e) is increased than in the previous work simulated in figure 4 . (f). When the number of nodes is increased in the routing zone of a node then the radius of the node is increases which decreases the throughput of network. This result shows that when the ratio of packet delivery and throughput is small, it means that mobility in the network is high when the number of nodes increases in the network. When the node's radius of zone is increased then it also increases routing load in the network. When the zone radius is kept low in range it increases the throughput, in which ZRP shows best performance. ZRP is commonly used for large networks, because it is giving better results when the size of the network is large. Table 4 shows the average throughput of ZRP. 


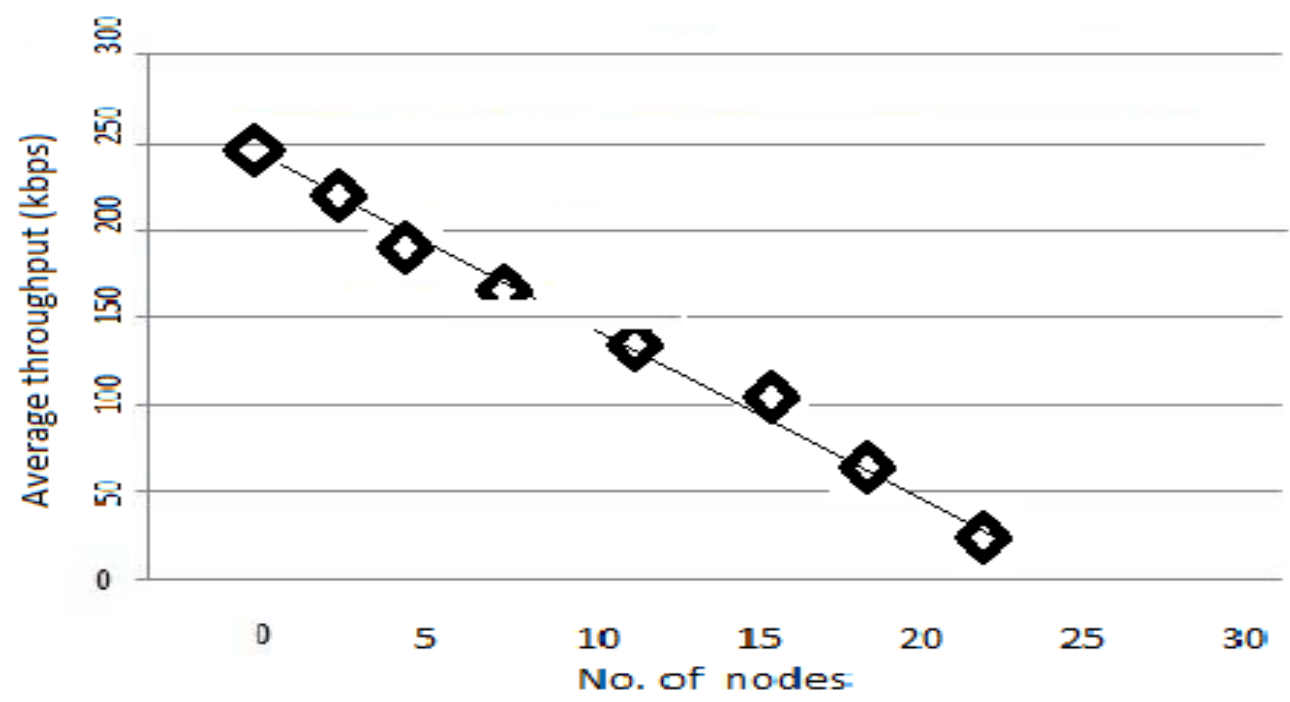

Figure 4. (e): Average Throughput of ZRP (Proposed Work)

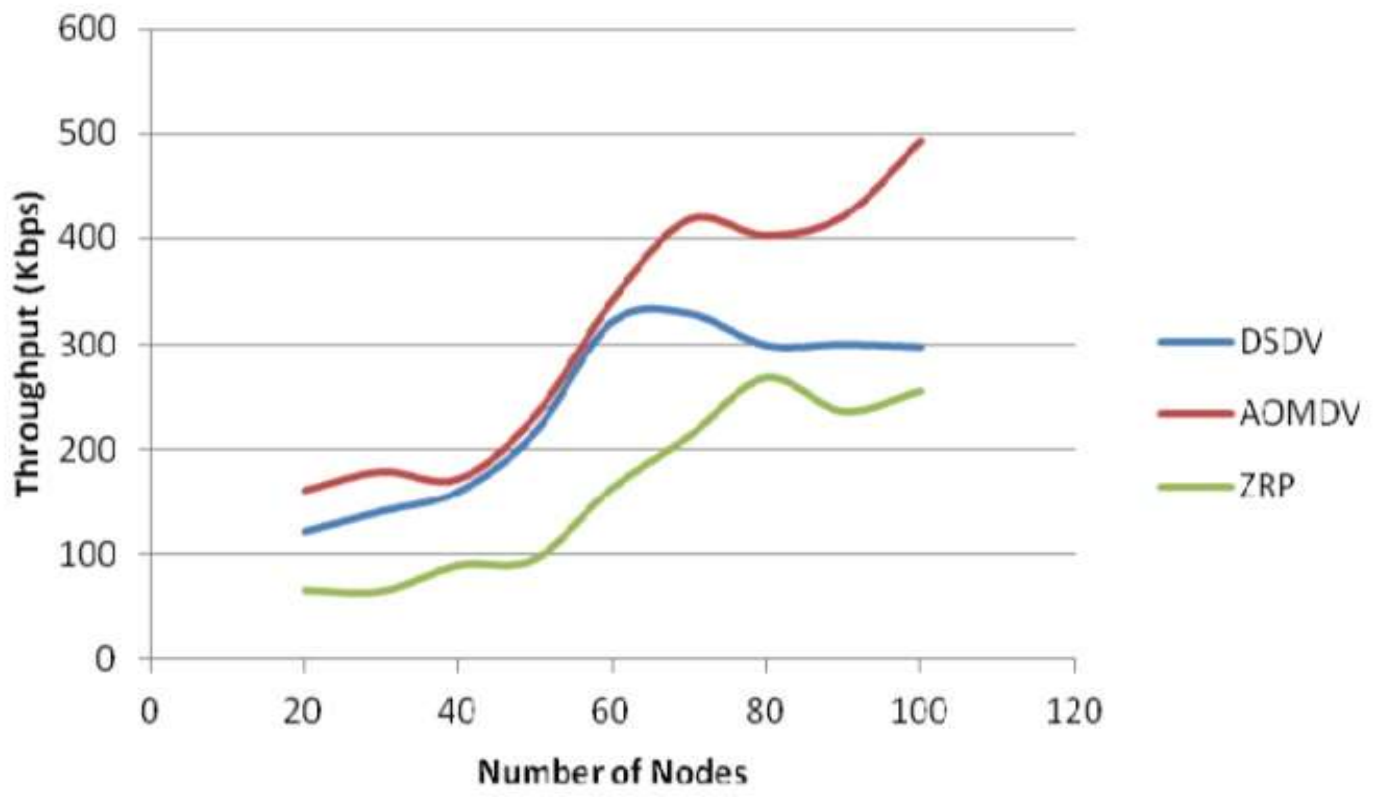

Figure 4. (f): Average Throughput of ZRP (Previous Work)

Table 4: Average Throughput of ZRP.

\begin{tabular}{|c|c|c|c|c|c|}
\hline ZRP & $\begin{array}{c}\text { Total } \\
\text { Routing } \\
\text { packets }\end{array}$ & $\begin{array}{c}\text { Received } \\
\text { data } \\
\text { packets }\end{array}$ & $\begin{array}{c}\text { Average } \\
\text { end to end } \\
\text { delay (sec) }\end{array}$ & $\begin{array}{c}\text { Dropped } \\
\text { from Queue }\end{array}$ & Throughput \\
\hline 5 nodes & $\mathbf{1 5 2 0 0}$ & $\mathbf{9 8 0 0}$ & $\mathbf{0 . 0 0 6}$ & $\mathbf{6 5}$ & $\mathbf{1 2 3 1 5 . 9 8}$ \\
\hline 10 nodes & $\mathbf{2 3 5 1 0}$ & $\mathbf{1 0 2 1 8}$ & $\mathbf{0 . 0 0 5 5}$ & $\mathbf{1 5 0}$ & $\mathbf{1 2 3 6 0 . 9}$ \\
\hline 15 nodes & $\mathbf{3 8 5 2 2}$ & $\mathbf{9 7 9 5}$ & $\mathbf{0 . 0 0 5 9}$ & $\mathbf{5 0}$ & $\mathbf{1 2 1 2 5 . 5 5}$ \\
\hline 20 nodes & $\mathbf{5 5 8 3 0}$ & $\mathbf{9 3 0 0}$ & $\mathbf{0 . 0 0 9 9}$ & $\mathbf{2 0 0}$ & $\mathbf{1 1 9 6 0 . 1 5}$ \\
\hline 25 nodes & $\mathbf{7 8 6 4 5}$ & $\mathbf{8 9 7 0}$ & $\mathbf{0 . 0 6 5}$ & $\mathbf{3 8 0}$ & $\mathbf{1 0 7 7 5 . 2 2}$ \\
\hline 30 nodes & $\mathbf{9 5 2 1 5}$ & $\mathbf{8 1 5 5}$ & $\mathbf{0 . 0 8 5}$ & $\mathbf{6 4 5}$ & $\mathbf{9 6 2 5 . 6 8}$ \\
\hline
\end{tabular}




\section{Conclusion and Future Work}

In this research paper the performance of Zone Routing Protocol (ZRP) in mobile Ad-hoc Network environment. Selective bordercasting technique is used to minimize flooding in the overlapping zones of the ZRP. ZRP is the combination of proactive and reactive Protocols. The Proactive part of the ZRP is used for routing inside the zone of the node while the reactive portion of ZRP is working outside of the routing zone. The outer node connected to other nodes of different zones. The evaluation criteria used in this research work consists of Packet Delivery Ratio, Average end-to-end delay and throughput, in which ZRP shows better performance and reduced flooding in our proposed scenario. The performance of the ZRP is depending upon the size of the network. For large network ZRP gives best performance and improve efficiency. The performance of the ZRP is also dependent on the Zone Radius. For smaller zone radius the PDF is high. When the data rate is high then it is very difficult task for ZRP to handle it efficiently because in this case the data rate of the transmitted data packets on the network is high, and as a result huge number of packets are lost during transmission. The performance of ZRP is improved with throughput by decreasing end to end delay of packets on the network. The Zone Routing Protocol (ZRP) provides a hybrid routing framework, in which each node keeps native routes within its zone using a proactive approach, while inter-zone communication is performed using a reactive approach. In order to enhance the performance of the reactive IERP and to minimize flooding used by all neighboring nodes which is drastically affects the available bandwidth required to be used by the broadcasting of the underlying protocol. As a future we will work on the zone size of the ZRP that plays a significant role in the performance of this protocol.

\section{REFERNCES}

[1] Ahmed, S., Bilal, M., \& Farooq, U. (2007, November). Performance Analysis of various routing strategies in Mobile Ad hoc Network using QualNet simulator. In Emerging Technologies, 2007. ICET 2007. International Conference on (pp. 62-67). IEEE.

[2] Ahmed, S. (2013). Performance Analysis of DSR and AODV Routing Protocols. International Journal of Computer Theory and Engineering, 5(3), 582.

[3] Annamalai, P. (2005). Comparative Performance Study of Standardized Ad-Hoc Routing Protocols and OSPF-MCDS (Doctoral dissertation, Virginia Polytechnic Institute and State University).

[4] Basagni, S., Conti, M., Giordano, S., \& Stojmenovic, I. (2003). Mobile ad hoc Networks.

[5] Buchegger, S., \& Le Boudec, J. Y. (2002, June). Performance analysis of the CONFIDANT protocol. In Proceedings of the 3rd ACM international symposium on Mobile ad hoc networking \& computing (pp. 226-236). ACM.

[6] Chlamtac, I., Conti, M., \& Liu, J. J. N. (2003). Mobile ad hoc networking: imperatives and challenges. Ad hoc networks, 1(1), 13-64.

[7] Clausen, T., Jacquet, P., Adjih, C., Laouiti, A., Minet, P., Muhlethaler, P., \& Viennot, L. (2003). Optimized link state routing protocol (OLSR).

[8] Broch, J., Johnson, D. B., \& Maltz, D. A. (1998). The dynamic source routing protocol for mobile ad hoc networks.

[9] Gantsou, D., Sondi, P., \& Hanafi, S. D. (2008). Revisiting multipoint relay selection in the optimized link state routing protocol. International Journal of Communication Networks and Distributed Systems, 2(1), 4-15.

[10] Gerla, M., Hong, X., Ma, L., \& Pei, G. (2002). Landmark routing protocol (LANMAR) for large scale ad hoc networks. Internet Engineering Task Force (IETF) draft, November.

[11] Giannoulis, S., Antonopoulos, C., Topalis, E., \& Koubias, S. (2005, September). ZRP versus DSR and TORA: A comprehensive survey on ZRP performance. In Emerging Technologies and Factory Automation, 2005. ETFA 2005. 10th IEEE Conference on (Vol. 1, pp. 8-pp). IEEE.

[12] Haas, Z. J., \& Pearlman, M. R. (2001). The performance of query control schemes for the zone routing protocol. IEEE/ACM Transactions on Networking (TON), 9(4), 427-438.

[13] Jacquet, P., Mühlethaler, P., Clausen, T., Laouiti, A., Qayyum, A., \& Viennot, L. (2001). Optimized link state routing protocol for ad hoc networks. In Multi Topic Conference, 2001. IEEE INMIC 2001. Technology for the 21st Century. Proceedings. IEEE International (pp. 62-68). IEEE. 
[14] Jain, N., \& Chaba, Y. (2014). Simulation based performance analysis of zone routing protocol in Manet. International Journal of Computer Applications,88(4).

[15] Johnson, D. B. (2003). The dynamic source routing protocol for mobile ad hoc networks. draft-ietf-manet-dsr-09. txt.

[16] Jorg, D. O. (2003). Performance comparison of MANET routing protocols in different network sizes. Computer Networks \& Distributed Systems.

[17] Loutfi, A., \& Elkoutbi, M. (2011, April). Evaluation and enhancement of ZRP performances. In Multimedia Computing and Systems (ICMCS), 2011 International Conference on (pp. 1-6). IEEE.

[18] ad hoc Networking, M. (1999). Routing Protocol Performance Issues and Evaluation Considerations (Vol. 2501). IETF RFC.

[19] Mittal, S., \& Kaur, P. (2009, December). Performance Comparison of AODV, DSR and ZRP Routing Protocols in MANET's. In 2009 International Conference on Advances in Computing, Control, and Telecommunication Technologies (pp. 165-168). IEEE.

[20] Murthy, C. S. R., \& Manoj, B. S. (2004). Ad Hoc Wireless Networks: Architectures and Protocols, Portable Documents. Pearson education.

[21] Pearlman, M. R., \& Haas, Z. J. (1999). Determining the optimal configuration for the zone routing protocol. Selected Areas in Communications, IEEE Journal on, 17(8), 1395-1414.

[22] Perkins, C. E., \& Bhagwat, P. (1994, October). Highly dynamic destination-sequenced distance-vector routing (DSDV) for mobile computers. In ACM SIGCOMM computer communication review (Vol. 24, No. 4, pp. 234-244). ACM.

[23] Royer, E. M., \& Toh, C. K. (1999). A review of current routing protocols for ad hoc mobile wireless networks. Personal Communications, IEEE, 6(2), 46-55.

[24] Schaumann, J. (2002). Analysis of the zone routing protocol. Course CS765, Stevens Institute of Technology Hoboken, New Jersey, USA, 8th December.

[25] Shafiq, Z., Mahmud, S. A., Khan, G. M., Sayyed, A., \& Al-Raweshidy, H. S. (2012, October). Zone Routing Protocol: How does it perform the other way round?. In ICT Convergence (ICTC), 2012 International Conference on (pp. 71-77). IEEE.

[26] Shrestha, A., \& Tekiner, F. (2009, December). On MANET routing protocols for mobility and scalability. In Parallel and Distributed Computing, Applications and Technologies, 2009 International Conference on (pp. 451-456). IEEE.

[27] Upadhyay, S., Joshi, P., Gandotra, N., \& Kumari, A. (2012). Comparison and performance analysis of reactive type DSR, AODV and proactive type DSDV routing protocol for wireless mobile ad-hoc networking, using NS-2 simulator.Journal of Engineering and Computer Innovations, 2(10), 36-47.

[28] Vetrivelan, N., \& Reddy, A. V. (2008, March). Performance Analysis of Three Routing Protocols for Varying MANET Size. In Proceedings of the International MultiConference of Engineers and Computer Scientists (Vol. 2, pp. 19-21).

[29] Webb, J. W. (2005). Analysis of Packet Flows in Simulated Ad Hoc Networks Using Standard Network Tools (Doctoral dissertation, UNIVERSITY OF CALIFORNIA SANTA CRUZ).

[30] Yi, J., Adnane, A., David, S., \& Parrein, B. (2011). Multipath optimized link state routing for mobile ad hoc networks. Ad Hoc Networks, 9(1), 28-47. 